\title{
Global Optimization for the Sum of Concave-Convex Ratios Problem
}

\author{
XueGang Zhou ${ }^{1,2}$ and JiHui Yang ${ }^{3}$ \\ ${ }^{1}$ School of Mathematics and Information Science, Key Laboratory of Mathematics and Interdisciplinary Sciences of Guangdong, \\ Higher Education Institutes, Guangzhou University, Guangzhou, Guangdong 510006, China \\ ${ }^{2}$ Department of Applied Mathematics, Guangdong University of Finance, Guangzhou, Guangdong 510521, China \\ ${ }^{3}$ College of Science, Shenyang Agricultural University, Shenyang, Liaoning 110866, China
}

Correspondence should be addressed to JiHui Yang; yangjihui@163.com

Received 29 January 2014; Revised 10 April 2014; Accepted 10 April 2014; Published 13 May 2014

Academic Editor: Jen-Chih Yao

Copyright (C) 2014 X. Zhou and J. Yang. This is an open access article distributed under the Creative Commons Attribution License, which permits unrestricted use, distribution, and reproduction in any medium, provided the original work is properly cited.

\begin{abstract}
This paper presents a branch and bound algorithm for globally solving the sum of concave-convex ratios problem (P) over a compact convex set. Firstly, the problem $(\mathrm{P})$ is converted to an equivalent problem $(\mathrm{P} 1)$. Then, the initial nonconvex programming problem is reduced to a sequence of convex programming problems by utilizing linearization technique. The proposed algorithm is convergent to a global optimal solution by means of the subsequent solutions of a series of convex programming problems. Some examples are given to illustrate the feasibility of the proposed algorithm.
\end{abstract}

\section{Introduction}

We consider the concave-convex ratios programming problems as follows:

$$
\text { (P) }\left\{\begin{aligned}
v=\max \quad f(x) & =\sum_{i=1}^{p} \frac{f_{i}(x)}{g_{i}(x)}, \\
\text { s.t. } \quad x \in X & =\left\{x \in R^{n} \mid A x \leqslant b\right\},
\end{aligned}\right.
$$

where $p \geqslant 2, f_{i}, i=1,2, \ldots, p$, are concave and differentiable functions defined on $R^{n}, g_{i}, i=1,2, \ldots, p$, are convex and differentiable functions defined on $R^{n}, X \subseteq R^{n}$ is a nonempty, compact convex set, and for each $i=1,2, \ldots, p, f_{i}(x)>0$ and $g_{i}(x)>0$ for all $x \in X$.

During the past years, various algorithms have been proposed for solving special cases of fractional programming problem. For instance, algorithmic and computational results for single ratio fractional programming can be found in $[1,2]$ and in the literature cited therein. At present, there exist a number of algorithms for globally solving sum of ratios problem in which the numerators and denominators are affine functions or the feasible region is a polyhedron [3-5]. To my knowledge, four algorithms have been proposed for solving the nonlinear sum of ratios problem [6-9]. Freund and Jarre [10] present a suitable interior-point approach for the solution of much more general problems with convex-concave ratios and convex constraints. Shen et al. [11] present a simplicial branch and duality bound algorithm for globally solving the sum of convex-convex ratios problem with nonconvex feasible region.

In this paper, we implement a branch and bound algorithm for globally solving problem $(\mathrm{P})$. First, although the branch and bound search involves rectangles defined in a space of dimension $3 p$, branching takes place in a space of only dimension $p$, where $p$ is the number of ratios in the objective function of problem (P). Second, all subproblems that must be solved to implement the algorithm are convex programming problems, each of which is guaranteed to have an optimal solution. Finally, some examples are given to show that the proposed method can treat all of the test problems in finding globally optimal solutions within a prespecified tolerance. The algorithms of this paper were motivated by the seminal works of [12], the generalized concave multiplicative programming problem.

The organization and content of this paper can be summarized as follows. In Section 2, we demonstrate how to convert problem $(\mathrm{P})$ into an equivalent problem (P1). By using the convex envelope of the bilinear function and the special characteristics of quadratic function, we will 
illustrate how to generate the convex relaxation program for problem (P1) in Section 3. In Section 4, the branch and bound algorithm for globally solving $(\mathrm{P})$ is presented. And convergence properties of the algorithm and computational considerations for implementing the algorithm are given. Some numerical examples are given to demonstrate the effectiveness of the proposed algorithm in Section 5. Some concluding remarks are given in Section 6.

\section{Equivalent Program}

To globally solve problem (P), the branch and bound algorithm globally solves a problem (P1) equivalent to problem (P). In this section, the following main work is to show how to convert problem $(\mathrm{P})$ into an equivalent nonconvex programming problem (P1).

Let $I=\{1,2, \ldots, p\}$. For each $i \in I$, let $h_{i}(x)=$ $\sqrt{f_{i}(x)} / g_{i}(x)$. Then, we have the following result.

Proposition 1. Let $X^{\prime}$ be an open set containing $X$ such that for each $i=1,2, \ldots, p, f_{i}(x)>0, g_{i}(x)>0$, for all $x \in X$. Then, for each $i=1,2, \ldots, p$, the function $h_{i}(x)$ is semistrictly quasiconcave on $X^{\prime}$.

Proof. For any $i \in I$, it is easy to show that the function $\sqrt{f_{i}(x)}$ is concave and differentiable on $X^{\prime}$. Since $g_{i}(x)$ is positive, convex, and differentiable on $X^{\prime}$, from Avriel et al. [13], this implies that $h_{i}(x)$ is semistrictly quasiconcave on $X^{\prime}$.

Proposition 2. Let $X^{\prime}$ be defined as in Proposition 1. For each $i \in I$, we consider the problem

$$
\left(P_{i}\right) \quad U_{i}^{0}=\max _{x \in X} h_{i}(x) .
$$

Then, any local maximum is also a global maximum of problem $\left(P_{i}\right)$.

Proof. Since $X$ is a convex set, the result follows directly from Proposition 1 and Theorem 3.37 of [13].

Therefore, $U_{i}^{0}$ can be found by any number of convex programming algorithms. Let $H^{0}=\left\{u \in R^{p} \mid 0 \leqslant u_{i} \leqslant\right.$ $\left.U_{i}^{0}, i \in I\right\}$. Then, $H^{0}$ is a full-dimensional rectangle in $R^{p}$. Let $\Phi: X \times H^{0} \rightarrow R$ be defined for each $(x, u) \in X \times H^{0}$ by

$$
\Phi(x, u)=\sum_{i \in I}\left[2 u_{i} \sqrt{f_{i}(x)}-u_{i}^{2}\left(g_{i}(x)\right)\right] .
$$

For any $u \in H^{0}$, define the problem $\left(S_{u}\right)$ by

$$
\left(S_{u}\right) \quad \varphi(u)=\max _{x \in X} \Phi(x, u) .
$$

Definition 3 (see [14]). Let $X$ and $Z$ be convex subsets of $R^{m}$ and $R^{q}$, respectively. A real-valued function $h$ defined on $X \times$ $Z$ is biconcave if, for each fixed $\bar{x} \in X, h(\bar{x}, z)$ is a concave function on $Z$ and, for each fixed $\bar{z} \in Z, h(x, \bar{z})$ is a concave function on $X$. The following result shows that, for every $u \in$ $H^{0}$, the value of $\varphi(u)$ can be determined by solving a convex program.
Lemma 4. The objective function $\Phi(x, u)$ of problem $\left(S_{u}\right)$ is biconcave on $X \times H^{0}$.

Proof. For each $i \in I$, let $H_{i}^{0}=\left\{u_{i} \in R \mid 0 \leqslant u_{i} \leqslant U_{i}^{0}\right\}$ and define $\Phi_{i}: X \times H_{i}^{0} \rightarrow R$ by $\Phi_{i}\left(x, u_{i}\right)=2 u_{i} \sqrt{f_{i}(x)}-$ $u_{i}^{2}\left(g_{i}(x)\right)$. Therefore, for every $x \in X$ and $u \in H^{0}$, we have $\Phi(x, u)=\sum_{i=1}^{p} \Phi_{i}(x, u)$. Notice that $X, H^{0}$ and $H_{i}^{0}(i \in I)$ are convex sets; then it will suffice to show that, for every $i \in I, \Phi_{i}$ is biconcave on $X \times H_{i}^{0}$. Given $\widehat{u}_{i} \in H_{i}^{0}$. Thus, for any $i \in I, \Phi_{i}\left(x, \widehat{u}_{i}\right)=2 \widehat{u}_{i} \sqrt{f_{i}(x)}-\widehat{u}_{i}^{2} g_{i}(x)$. For all $x \in X$, $2 \widehat{u}_{i} \sqrt{f_{i}(x)}(i=1,2, \ldots, p)$ are concave, since the function $f_{i}(x)(i=1,2, \ldots, p)$ is concave and $\widehat{u}_{i} \geqslant 0, f_{i}(x)>0$ $(i=1,2, \ldots, p)$. Because the function $g_{i}(x)$ is positive convex function on $X$ and $\widehat{u}_{i} \geqslant 0,-\widehat{u}_{i}^{2} g_{i}(x)$ is also concave on $X$. Therefore, it follows that $\Phi_{i}\left(x, \widehat{u}_{i}\right)$ is a concave function on $x$.

Now, let $\hat{x} \in X$ be a fixed vector. For all $u_{i} \in H_{i}^{0}$, $\Phi_{i}\left(\widehat{x}, u_{i}\right)=2 u_{i} \sqrt{f_{i}(\widehat{x})}-u_{i}^{2} g_{i}(\widehat{x})$. Since $g_{i}(\widehat{x})>0$, it follows easily that $\Phi_{i}\left(\hat{x}, u_{i}\right)$ is a concave function on $H_{i}^{0}$. The proof is complete.

We now define the problem (P1) by

$$
\text { (P1) } \quad v 1=\max _{u \in U^{0}} \varphi(u)=\max _{u \in U^{0}} \max _{x \in X} \Phi(x, u) \text {. }
$$

Theorem 5. The problem $(P)$ is equivalent to the problem $(P 1)$ in the following sense: if $u^{*}$ is an optimal solution to the problem $(P 1)$ and if $x^{*}$ is a corresponding optimal solution of problem $\left(S_{u}\right)$ with $u=u^{*}$, then $x^{*}$ is an optimal solution for problem $(P)$. Moreover, the following relations hold:

$$
\begin{gathered}
u^{*}=\frac{\sqrt{f_{i}\left(x^{*}\right)}}{g_{i}\left(x^{*}\right)}, \quad i \in I, \\
v 1=\varphi\left(u^{*}\right)=f\left(x^{*}\right)=v .
\end{gathered}
$$

Conversely, if $x^{*}$ is an optimal solution of problem $(P)$, the value $u^{*}$ deduced from relation (6) corresponds to an optimal solution of problem $(P 1)$ and relation $(7)$ holds.

Proof. Let $u^{*}$ be a global optimal solution for problem (P1), and let $x^{*}$ solve problem $\left(S_{u}\right)$ with $u=u^{*}$; then $\left(x^{*}, u^{*}\right) \in$ $X \times H^{0}$. Then,

$$
\begin{aligned}
\varphi\left(u^{*}\right) & =\max _{u \in H^{0}} \varphi(u)=\max _{x \in X} \sum_{i \in I}\left[2 u_{i}^{*} \sqrt{f_{i}(x)}-\left(u_{i}^{*}\right)^{2} g_{i}(x)\right] \\
& =\sum_{i \in I}\left[2 u_{i}^{*} \sqrt{f_{i}\left(x^{*}\right)}-\left(u_{i}^{*}\right)^{2} g_{i}\left(x^{*}\right)\right] .
\end{aligned}
$$

It follows from the definition of $\varphi$ and (10) that $\left(x^{*}, u^{*}\right)$ is a global optimal solution to the problem

$$
\text { (P2) } \max _{x \in X, u \in H^{0}} \Phi(x, u) \text {. }
$$

Therefore, $u^{*}$ is global optimal solution to the problem

$$
\max _{u \in H^{0}} \Phi\left(x^{*}, u\right)
$$


For each $i \in I$, define $r_{i}: R \rightarrow R$ for each $u_{i} \in R$ by

$$
r_{i}\left(u_{i}\right)=2 u_{i} \sqrt{f_{i}\left(x^{*}\right)}-u_{i}^{2} g_{i}\left(x^{*}\right) .
$$

Then, for all $i \in I$, since $g_{i}(x)>0, r_{i}\left(u_{i}\right)$ is a strictly concave function, and the maximum of $r_{i}\left(u_{i}\right)$ over $u_{i} \in R$ is attained uniquely at $u_{i}^{0}=\sqrt{f_{i}\left(x^{*}\right)} / g_{i}\left(x^{*}\right)$. By definition of $H^{0},\left(u^{0}\right)^{T} \triangleq$ $\left(u_{1}^{0}, u_{2}^{0}, \ldots, u_{p}^{0}\right) \in H^{0}$. The previous two statements imply that $u^{0}$ is the unique optimal solution to (12). Therefore, $u^{*}=u^{0}$ and the objective function value in (12) of $u^{*}$ is

$$
\begin{aligned}
\Phi\left(x^{*}, u^{*}\right) & =\sum_{i \in I}\left[2 u_{i}^{*} \sqrt{f_{i}\left(x^{*}\right)}-\left(u_{i}^{*}\right)^{2} g_{i}\left(x^{*}\right)\right] \\
& =\sum_{i \in I}\left[2 \frac{f_{i}\left(x^{*}\right)}{g_{i}\left(x^{*}\right)}-\frac{f_{i}\left(x^{*}\right)}{g_{i}\left(x^{*}\right)}\right]=\sum_{i \in I} \frac{f_{i}\left(x^{*}\right)}{g_{i}\left(x^{*}\right)} \\
& =f\left(x^{*}\right) .
\end{aligned}
$$

So, $f\left(x^{*}\right)$ is also the objective function value of $\left(x^{*}, u^{*}\right)$ in problem (P2). Assume that there exist some $x \in X$ such that $f(x)>f\left(x^{*}\right)$. Let $u_{i}=\left(\sqrt{f_{i}(x)} / g_{i}(x)\right)(i \in I)$. Then, $u^{T}=\left(u_{1}, u_{2}, \ldots, u_{p}\right) \in H^{0}$, and the objective function value of $(x, u)$ in problem $(\mathrm{P} 2)$ is

$$
\begin{aligned}
\Phi(x, u) & =\sum_{i \in I}\left[2 u_{i} \sqrt{f_{i}(x)}-\left(u_{i}\right)^{2} g_{i}(x)\right] \\
& =\sum_{i \in I} \frac{f_{i}(x)}{g_{i}(x)}=f(x) .
\end{aligned}
$$

It follows from $f(x)>f\left(x^{*}\right)$ and (16) that $\left(x^{*}, u^{*}\right)$ is not a global optimal solution to problem (P2), which is a contradiction. This implies that, for all $x \in X, f(x) \leqslant f\left(x^{*}\right)$; that is, $x^{*}$ is a global optimal solution for problem (P). From (10) and (16), $\varphi\left(u^{*}\right)=f\left(x^{*}\right)$. Since $f\left(x^{*}\right)=v$, this completes the proof of the first statement of the theorem.

Now suppose that $x^{*}$ is a global optimal solution for problem (P). Then, $x^{*} \in X$ and $f_{i}\left(x^{*}\right)>0, g_{i}\left(x^{*}\right)>0$ for all $i \in I$. We compute $\left(u^{*}\right)^{T} \triangleq\left(u_{1}^{*}, u_{2}^{*}, \ldots, u_{p}^{*}\right) \in H^{0}$ by (7). From the definition of $\varphi$,

$$
\begin{aligned}
\varphi\left(u^{*}\right) & =\max _{x \in X}\left\{\sum_{i \in I}\left[2 u_{i}^{*} \sqrt{f_{i}(x)}-\left(u_{i}^{*}\right)^{2} g_{i}(x)\right]\right\} \\
& \geqslant \sum_{i \in I}\left[2 u_{i}^{*} \sqrt{f_{i}\left(x^{*}\right)}-\left(u_{i}^{*}\right)^{2} g_{i}\left(x^{*}\right)\right] \\
& =\sum_{i \in I} \frac{f_{i}\left(x^{*}\right)}{g_{i}\left(x^{*}\right)}=f\left(x^{*}\right) .
\end{aligned}
$$

Suppose that $\varphi\left(u^{1}\right)>\varphi\left(u^{*}\right)$ for some $u^{1} \in U^{0}$ and $x^{1}$ is a corresponding optimal solution of problem $\left(S_{u}\right)$ with $u=u^{1}$. By the first part of the theorem, this implies that $f\left(u^{1}\right)>$ $f\left(x^{*}\right)=v 1$. Therefore, since $u^{*} \in H^{0}, \varphi\left(u^{*}\right)=v 1$, and $u^{*}$ is a global optimal solution for problem (P1).

\section{Relaxation Problem for Problem (P1)}

Let $H=\left\{u \in R^{p} \mid L \leqslant u \leqslant U\right\}$ denote $H^{0}$ or a subrectangle of $H^{0}$ that is generated by the branch and bound algorithm, where $L, U \in R^{p}$ and $0 \leqslant L_{i}<U_{i}$ for all $i \in I$. We consider the following problem:

$$
(\mathrm{P} 1(H)) \quad \max _{u \in H} \varphi(u)
$$

For each $i \in I$, let $\bar{t}_{i}=\max _{x \in X} \sqrt{f_{i}(x)}$, and let $\bar{s}_{i}$ satisfy

$$
\bar{s}_{i} \geqslant \max _{x \in X} g_{i}(x)>0 .
$$

Since, for every $i \in I, c_{i}(x)=\sqrt{f_{i}(x)}$ is a concave function on $X$, then, for each $i \in I, \bar{t}_{i}$ can be found by solving a convex programming problem. For each $i \in I, \bar{s}_{i}$ can be chosen to be a sufficiently large positive number.

Now, consider the function $G: H^{0} \rightarrow R$ which is given for any $u \in H^{0}$ by

$$
\begin{aligned}
(\mathrm{P}(u)) \quad G(u)=\max & \sum_{i=1}^{p}\left[2 u_{i} t_{i}-u_{i}^{2} s_{i}\right], \\
\text { s.t. } \quad & t_{i}-\sqrt{f_{i}(x)} \leqslant 0, \quad i \in I, \\
& -s_{i}+g_{i}(x) \leqslant 0, \quad i \in I, \\
& 0 \leqslant t_{i} \leqslant \bar{t}_{i}, \quad 0 \leqslant s_{i} \leqslant \bar{s}_{i}, \quad i \in I, \\
& x \in X .
\end{aligned}
$$

Theorem 6. For each $u \in H^{0}, \varphi(u)=G(u)$. Moreover, if $u \in$ $H^{0}$ and $\left(x^{*}, t^{*}, s^{*}\right)$ is an optimal solution to problem $(P(u))$, then $\varphi(u)=\sum_{i \in I}\left[2 u_{i} \sqrt{f_{i}\left(x^{*}\right)}-u_{i}^{2} g_{i}\left(x^{*}\right)\right]$.

Proof. Let $u \in H^{0}$. On the basis of the definition $\varphi$, for some $\widehat{x} \in X$,

$$
\varphi(u)=\sum_{i \in I}\left[2 u_{i} \sqrt{f_{i}(\widehat{x})}-u_{i}^{2} g_{i}(\widehat{x})\right]
$$

For every $i \in I$, let

$$
\widehat{t}_{i}=\sqrt{f_{i}(\widehat{x})}, \quad \widehat{s}_{i}=g_{i}(\widehat{x}) .
$$

Assume that $\hat{t}^{T}=\left(\widehat{t}_{1}, \widehat{t}_{2}, \ldots, \widehat{t}_{p}\right)$ and $\widehat{s}^{T}=\left(\widehat{s}_{1}, \widehat{s}_{2}, \ldots, \widehat{s}_{p}\right)$. So, $(\widehat{x}, \widehat{t}, \widehat{s})$ is a feasible solution to problem $(\mathrm{P}(u))$ with objective function value

$$
\sum_{i \in I}\left[2 u_{i} \widehat{t}_{i}-u_{i}^{2} \widehat{s}_{i}\right]=\varphi(u)
$$


where the equation follows from (18) and (19). Then, we have $G(u) \geqslant \varphi(u)$. Thus, in order to show the first result in theorem, we only show that $G(u)>\varphi(u)$ does not hold.

Assume that $G(u)>\varphi(u)$. Based on (18), there exists some feasible solution $(x, t, s)$ for problem $(\mathrm{P}(u))$ such that

$$
\begin{aligned}
\sum_{i \in I}\left[2 u_{i} t_{i}-u_{i}^{2} s_{i}\right] & >\varphi(u) \\
& =\sum_{i \in I}\left[2 u_{i} \sqrt{f_{i}(\widehat{x})}-u_{i}^{2} g_{i}(\widehat{x})\right] .
\end{aligned}
$$

According to definition of $0 \leqslant t_{i} \leqslant \sqrt{f_{i}(x)}$ and $0 \leqslant g_{i}(x) \leqslant s_{i}$ in the problem $(\mathrm{P}(u))$, for all $i \in I$ and $u \geqslant 0$, we have

$$
\sum_{i \in I}\left[2 u_{i} \sqrt{f_{i}(x)}-u_{i}^{2} g_{i}(x)\right] \geqslant \sum_{i \in I}\left[2 u_{i} t_{i}-u_{i}^{2} s_{i}\right] .
$$

From (18), (21), and (22), we obtain

$$
\sum_{i \in I}\left[2 u_{i} \sqrt{f_{i}(x)}-u_{i}^{2} g_{i}(x)\right]>\varphi(u)
$$

Since $x \in X$, this is a contradiction with the definition of $\varphi(u)$. Therefore, the assumption that $G(u)>\varphi(u)$ is false. This implies that $\varphi(u)=G(u)$, for each $u \in H^{0}$.

Now, we show the second part of the theorem. Let $\left(x^{*}, t^{*}, s^{*}\right)$ be an optimal solution to problem $(\mathrm{P}(u))$. Then, for each $i \in I, 0<t^{*} \leqslant \sqrt{f_{i}\left(x^{*}\right)}, s^{*} \geqslant g_{i}\left(x^{*}\right)>0$. So, we have

$$
\begin{aligned}
G(u) & =\sum_{i \in I}\left[2 u_{i} t_{i}^{*}-u_{i}^{2} s_{i}^{*}\right] \\
& \leqslant \sum_{i \in I}\left[2 u_{i} \sqrt{f_{i}\left(x^{*}\right)}-u_{i}^{2} g_{i}\left(x^{*}\right)\right] .
\end{aligned}
$$

Let $\widehat{t}^{T}=\left(\widehat{t}_{1}, \widehat{t}_{2}, \ldots, \widehat{t}_{p}\right)$ and $\widehat{s}^{T}=\left(\widehat{s}_{1}, \widehat{s}_{2}, \ldots, \widehat{s}_{p}\right)$, where, for each $i \in I$,

$$
\widehat{t}_{i}=\sqrt{f_{i}(\widehat{x})}, \quad \widehat{s}_{i}=g_{i}(\widehat{x})
$$

Then, $\left(x^{*}, \widehat{t}, \widehat{s}\right)$ is a feasible solution for problem $(\mathrm{P}(u))$, so that, by definition of $G(u)$,

$$
G(u) \geqslant \sum_{i \in I}\left[2 u_{i} \sqrt{f_{i}\left(x^{*}\right)}-u_{i}^{2} g_{i}\left(x^{*}\right)\right]
$$

From (24) and (26), it follows that

$$
\varphi(u)=\sum_{i \in I}\left[2 u_{i} \sqrt{f_{i}\left(x^{*}\right)}-u_{i}^{2} g_{i}\left(x^{*}\right)\right]
$$

since $\varphi(u)=G(u)$.
It follows form Theorem 6 that problem $(\mathrm{P} 1(H))$ has the same optimal value $v(H)$ with the following problem:

$$
\begin{array}{ll}
(\operatorname{PE} 1(M)) \quad \max \quad \sum_{i=1}^{p}\left[2 u_{i} t_{i}-u_{i}^{2} s_{i}\right], & \\
\text { s.t. } \quad & t_{i}-\sqrt{f_{i}(x)} \leqslant 0, \quad i \in I, \\
& -s_{i}+g_{i}(x) \leqslant 0, \quad i \in I, \\
& (t, s, u) \in M,
\end{array}
$$$$
x \in X,
$$

where $M=\left\{(t, s, u) \in R^{3 p} \mid 0 \leqslant t_{i} \leqslant \bar{t}_{i}, 0 \leqslant s_{i} \leqslant \bar{s}_{i}, L_{i} \leqslant\right.$ $\left.u_{i} \leqslant U_{i}, i \in I\right\}$.

In order to construct relaxation problem for problem (PE1), we must use the concept of a concave envelope, which may be defined as follows.

Definition 7 (see [15]). Let $M \subseteq R^{q}$ be a compact, convex set, and let $f: M \rightarrow R$ be upper semicontinuous on $M$. Then, $f^{M}: M \rightarrow R$ is called the concave envelope of $f$ on $M$ when

(i) $f^{M}(x)$ is a concave function on $M$,

(ii) $f^{M}(x) \leqslant f(x)$ for all $x \in M$,

(iii) there is no function $w(x)$ satisfying (i) and (ii) such that $w(\bar{x})<f^{M}(\bar{x})$ for some point $\bar{x} \in M$.

The convex envelope of a function $f$ on $M$ is defined in a similar manner.

Let, for each $i \in I, M_{i}=\left\{\left(u_{i}, t_{i}, s_{i}\right) \in R^{3} \mid L_{i} \leqslant u_{i} \leqslant\right.$ $\left.U_{i}, 0 \leqslant t_{i} \leqslant \bar{t}, 0 \leqslant s_{i} \leqslant \bar{s}\right\}$. Then, for each $i \in I, 0 \leqslant L_{i}<U_{i}$, and the concave envelope of $q_{i}^{M_{i}}\left(u_{i}\right)$ of the quadratic function $q_{i}\left(u_{i}\right)=u_{i}^{2}$ is given by

$$
q_{i}^{M_{i}}\left(u_{i}\right)=K_{i}\left(u_{i}-L_{i}\right)+L_{i}^{2}
$$

where $K_{i}=L_{i}+U_{i}$. Let $\underline{q}_{i}\left(u_{i}\right)$ represent the linear lower bounding function of $q_{i}\left(u_{i}\right)$ over the interval $L_{i} \leqslant u_{i} \leqslant U_{i}$. Then, by the convexity of the function $q_{i}$, the function $\underline{q}_{i}\left(u_{i}\right)$ is given as follows:

$$
\underline{q}_{i}\left(u_{i}\right)=K_{i}\left(u_{i}-\frac{1}{4} K_{i}\right)
$$

Lemma 8. Consider the functions $q_{i}\left(u_{i}\right), \underline{q}_{i}\left(u_{i}\right)$, and $q_{i}^{M_{i}}\left(u_{i}\right)$ for any $u_{i} \in H_{i}=\left[L_{i}, U_{i}\right]$, where $i=1,2, \ldots, p$. Then, the following two statements are valid.

(i) $q_{i}^{M_{i}}\left(u_{i}\right)$ is an affine concave envelope of $q_{i}\left(u_{i}\right)$ over $M_{i}$, and $q_{i}\left(u_{i}\right)$ is an affine function corresponding to a supporting hyperplane of the graph of $q_{i}\left(u_{i}\right)$ over $M_{i}$, which is parallel to $q_{i}^{M_{i}}\left(u_{i}\right)$. Moreover, we have

$$
\underline{q}_{i}\left(u_{i}\right) \leqslant q_{i}\left(u_{i}\right) \leqslant q_{i}^{M_{i}}\left(u_{i}\right), \quad \forall u_{i} \in H_{i} ; i=1,2, \ldots, p .
$$


(ii) When $\omega_{i}=U_{i}-L_{i} \rightarrow 0$, the differences $\Delta_{i}^{1}=q_{i}^{M_{i}}-q_{i}$ and $\Delta_{j}^{2}=q_{i}-\underline{q}_{i}$ satisfy

$$
\max _{u_{i} \in H_{i}} \Delta_{i}^{1}=\max _{u_{i} \in H_{i}} \Delta_{i}^{2}=\frac{1}{4}\left(U_{i}-L_{i}\right) \longrightarrow 0 .
$$

Proof. Consider the following:

(i) obviously,

(ii) since the $\Delta_{i}^{1}$ is a concave function about $u_{i}$ for any $u_{i} \in H_{i}, \Delta_{j}^{1}$ can attain the maximum $\Delta_{i \text {, max }}^{1}$ at the point $u_{i}^{*}=(1 / 2) K_{i}$. Thus, it is not difficult to have

$$
\Delta_{i, \max }^{1}=\frac{1}{4}\left(U_{i}-L_{i}\right) .
$$

On the other hand, since the $\Delta_{i}^{2}$ is a convex function about $u_{i}$ for any $u_{i} \in H_{i}, \Delta_{i}^{1}$ can attain the maximum $\Delta_{i \text {, max }}^{2}$ at the point $U_{i}$ or $L_{i}$. Thus,

$$
\Delta_{i, \max }^{2}=\frac{1}{4}\left(U_{i}-L_{i}\right) .
$$

Obviously, when $\omega_{i}=U_{i}-L_{i} \rightarrow 0$,

$$
\max _{u_{i} \in H_{i}} \Delta_{i}^{1}=\max _{u_{i} \in H_{i}} \Delta_{i}^{2}=\frac{1}{4}\left(U_{i}-L_{i}\right) \longrightarrow 0 .
$$

This completes the proof.

Therefore, for all $\left(u_{i}, t_{i}, s_{i}\right) \in M_{i}$, we can obtain

$$
\begin{aligned}
\sum_{i=1}^{p}\left[2 u_{i} t_{i}-q_{i}^{M_{i}}\left(u_{i}\right) s_{i}\right] & \leqslant \sum_{i=1}^{p}\left[2 u_{i} t_{i}-u_{i}^{2} s_{i}\right] \\
& \leqslant \sum_{i=1}^{p}\left[2 u_{i} t_{i}-\underline{q}_{i}\left(u_{i}\right) s_{i}\right],
\end{aligned}
$$

that is,

$$
\begin{aligned}
& \sum_{i=1}^{p}\left[2 u_{i} t_{i}-K_{i} u_{i} s_{i}+\left(K_{i} L_{i}-L_{i}^{2}\right) s_{i}\right] \\
& \leqslant \sum_{i=1}^{p}\left[2 u_{i} t_{i}-u_{i}^{2} s_{i}\right] \\
& \leqslant \sum_{i=1}^{p}\left[2 u_{i} t_{i}-K_{i} u_{i} s_{i}+\frac{1}{4} K_{i}^{2} s_{i}\right] .
\end{aligned}
$$

For each $i \in I, 0 \leqslant L_{i} \leqslant U_{i}$, and, from Benson [16], the concave envelope of $h_{i}^{M_{i}}\left(u_{i}, t_{i}\right)$ of the bilinear functions $h_{i}\left(u_{i}, t_{i}\right)$ is given for each $\left(u_{i}, t_{i}, s_{i}\right) \in M_{i}$ by

$$
h_{i}^{M_{i}}\left(u_{i}, t_{i}\right)=\min \left\{\bar{t}_{i} u_{i}+L_{i} t_{i}-\bar{t}_{i} L_{i}, U_{i} t_{i}\right\},
$$

and the convex envelope of $h_{i M_{i}}\left(u_{i}, s_{i}\right)$ of the bilinear functions $h_{i}\left(u_{i}, s_{i}\right)$ is given for each $\left(u_{i}, t_{i}, s_{i}\right) \in M_{i}$ by

$$
h_{i M_{i}}\left(u_{i}, s_{i}\right)=\max \left\{L_{i} s_{i}, \bar{s}_{i} u_{i}+U_{i} s_{i}-\bar{s}_{i} U_{i}\right\} .
$$

We now define the following problem:

$\operatorname{PR} 1(M)$

$$
\begin{array}{ll}
\mathrm{UB}(M)=\max & \sum_{i=1}^{p}\left[2 r_{i}-K_{i} z_{i}+\frac{1}{4} K_{i}^{2} s_{i}\right] \\
\text { s.t. } \quad & r_{i} \leqslant \bar{t}_{i} u_{i}+L_{i} t_{i}-\bar{t}_{i} L_{i}, \quad r_{i} \leqslant U_{i} t_{i} \\
& z_{i} \geqslant \bar{s}_{i} u_{i}+U_{i} s_{i}-\bar{s}_{i} U_{i}, \quad z_{i} \geqslant L_{i} s_{i}, \\
& t_{i}-\sqrt{f_{i}(x)} \leqslant 0, \\
& -s_{i}+g_{i}(x) \leqslant 0, \\
& (t, s, u) \in M, \quad r_{i} \geqslant 0, \quad z_{i} \geqslant 0, \\
& x \in X, \quad i \in I .
\end{array}
$$

Notice that the optimal value $\mathrm{UB}(M)$ of problem $\operatorname{PR} 1(M)$ satisfies $\mathrm{UB}(M) \geqslant v(H)$. It is also easy to see that the feasible region of problem PR1 $(M)$ is a nonempty compact set. Since the objective function of problem PR1 $(M)$ is affine function over this set, problem PR1 $(M)$ always has an optimal solution.

\section{Algorithm and Convergence}

To globally solve problem (P1), the algorithm to be presented uses a branch and bound approach. There are three fundamental processes in the algorithm: a branching process, a lower bounding process, and an upper bounding process.

4.1. Branching Rule. The algorithm performs a branching process in $R^{p}$ that iteratively subdivides the $p$-dimensional rectangle $H^{0}$ of problem (P1) into smaller rectangles that are also of dimension $p$. The branch and bound approach is based on partitioning the set $H$ into subrectangles, each concerned with a node of the branch and bound tree, and each node is associated with a relaxation linear subproblem on each subrectangle. These subrectangles are obtained by the branching process, which helps the branch and bound procedure identify a location in the feasible region of problem (P1) that contains a global optimal solution to the problem.

During each iteration of the algorithm, the branching process creates a more refined partition of a portion of $H^{1}=$ $H$ that cannot yet be excluded from consideration in the search for a global optimal solution for problem $\left(\mathrm{P} 1\left(H^{1}\right)\right)$. The initial partition $Q_{1}$ consists simply of $H^{1}$, since at the beginning of the branch and bound procedure, no portion of $H^{1}$ can as yet be excluded from consideration.

During iteration $k$ of the algorithm, $k \geqslant 1$, the branching process is used to help create a new partition $Q_{k+1}$. First, a screening procedure is used to remove any rectangle $H^{k}$ from $Q_{k}$ that $H^{k}$ can, at this point of the search, be excluded from further consideration, and $Q_{k+1}$ is temporarily set equal to the set of rectangles that remain. Later in iteration $k$, a rectangle $H^{k}$ in $Q_{k+1}$ is identified for further examination. 
The branching process is then evoked to subdivide $H^{k}$ into two subrectangles $H^{2 k}, H^{2 k+1}$. This subdivision is accomplished by a process called rectangular bisection.

Consider any node subproblem identified by the subrectangle $\widehat{H}$, where $\widehat{H}$ is defined as before. The branching rule is as follows [17].

Step 1. Let $U_{j}^{k-1}-L_{j}^{k-1}=\max _{i \in I}\left\{U_{i}^{k-1}-L_{i}^{k-1}\right\}$.

Step 2. Let $v_{j}$ satisfy $\min \left\{U_{j}^{k-1}-v_{j}, v_{j}-L_{j}^{k-1}\right\}=(1 / 2)\left(U_{j}^{k-1}-\right.$ $\left.L_{j}^{k-1}\right)$.

Step 3. Let

$$
\begin{aligned}
& H_{1}^{k-1} \\
& \quad=\left\{u \in \mathrm{R}^{N} \mid L_{j}^{k-1} \leqslant u_{j} \leqslant v_{j}, L_{i}^{k-1} \leqslant u_{i} \leqslant U_{j}^{k-1}, i \neq j\right\}, \\
& H_{2}^{k-1} \\
& \quad=\left\{u \in \mathrm{R}^{N} \mid v_{j} \leqslant u_{j} \leqslant U_{j}^{k-1}, L_{i}^{k-1} \leqslant u_{i} \leqslant U_{i}^{k-1}, i \neq j\right\} .
\end{aligned}
$$

The new partition $Q^{k}$ of the portion of $H^{0}$ remaining under consideration is then given by $Q^{k}=Q^{k-1}$ । $\left\{H^{k-1}\right\} \bigcup\left\{H_{1}^{k-1}, H_{2}^{k-1}\right\}$.

4.2. Lower Bound and Upper Bound. The second fundamental process of the algorithm is the upper bounding process. For each rectangle $H \in R^{p}$ created by the branching process, this process gives an upper bound $\mathrm{UB}(H)$ for the optimal value $v(H)$ of the problem $(\mathrm{P} 1(\mathrm{H}))$, that is,

$$
(\mathrm{P} 1(\mathrm{H})) \max _{u \in H} \varphi(u) .
$$

For each rectangle $H$ created by the branching process, from (40), $\mathrm{UB}(H)$ is found by solving a single convex program $(\operatorname{PR} 1(H))$.

During each iteration $k \geqslant 0$, the upper bounding process computes an upper bound for the optimal value $v 1$ of problem (P1). For each $k \geqslant 0$, this upper bound $\mathrm{UB}_{k}$ is given by

$$
\mathrm{UB}_{k}=\max \left\{\mathrm{UB}(H) \mid H \in Q^{k}\right\} .
$$

The lower bounding process is the third fundamental process of the branch and bound algorithm. In each iteration of the algorithm, this process finds a lower bound for $v 1$. For each $k \geqslant 0$, this lower bound $\mathrm{LB}_{k}$ is given by

$$
\mathrm{LB}_{k}=\varphi\left(\widehat{u}^{k}\right)
$$

where $\widehat{u}^{k}$ is the incumbent feasible solution for problem (P1); that is, among all of optimal solutions $(r, z, t, s, u, x)$ for problems of the form (PR1 $(H))$ found through iteration $k$, $u=\widehat{u}^{k}$ achieves the largest value of $\varphi$.

4.3. Branch and Bound Algorithm. Based on the results and algorithmic processes discussed in this section, the basic steps of the proposed global optimization are summarized in the following.

Step 0 (initialization). (i) Determine an optimal solution $\left(r^{0}, z^{0}, t^{0}, s^{0}, u^{0}, x^{0}\right)$ and the optimal value $\mathrm{UB}\left(H^{0}\right)$ to problem $\operatorname{PR} 1\left(H^{0}\right)$. Set $\mathrm{UB}_{0}=\mathrm{UB}\left(H^{0}\right), \mathrm{LB}_{0}=\varphi\left(u^{0}\right)$, and $\widehat{u}^{0}=u^{0}$. (ii) Set $Q^{0}=\left\{H^{0}\right\}$ and $k=1$, and go to iteration $k$.

\section{Iteration $k$.}

Step k.1. If $\mathrm{UB}_{k-1}=\mathrm{LB}_{k-1}$, then terminate. $\widehat{u}^{k-1}$ is a global optimal solution for problem (P1), and $v 1=\mathrm{LB}_{k-1}$. Then, we can solve problem $(\mathrm{P})$ on the basis of problem $\left(S_{u}\right)$ with $u=\widehat{u}^{k-1}$. If $\mathrm{UB}_{k-1} \neq \mathrm{LB}_{k-1}$, continue.

Step k.2. Subdivide $H^{k-1}$ into two rectangles $H_{1}^{k-1}$ and $H_{2}^{k-1}$ via the rectangular bisection.

Step k.3. For each $i=1,2$, find an optimal solution $\left(r^{i, k-1}, z^{i, k-1}, t^{i, k-1}, s^{i, k-1}, u^{i, k-1}, x^{i, k-1}\right)$ and the optimal value $\mathrm{UB}\left(H_{i}^{k-1}\right)$ to problem $\operatorname{PR} 1\left(H_{i}^{k-1}\right)$.

Step k.4. Set $\operatorname{LB}_{k}=\max \left\{\varphi\left(\widehat{u}^{k-1}\right), \varphi\left(u^{1, k-1}\right), \varphi\left(u^{2, k-1}\right)\right\}$, and choose $\widehat{u}^{k}$ so that $\mathrm{LB}_{k}=\varphi\left(\widehat{u}^{k}\right)$.

Step k.5. Set $Q_{k}=\left\{Q_{k-1} \backslash\left\{H^{k-1}\right\}\right\} \bigcup\left\{H_{1}^{k-1}, H_{2}^{k-1}\right\}$.

Step k.6. Delete from $Q^{k}$ all rectangles $H$ such that $\mathrm{UB}(H) \leqslant$ $\mathrm{LB}_{k}$.

Step k.7. If $Q^{k}=\emptyset$, set $\mathrm{UB}_{k}=\mathrm{LB}_{k}$, set $k=k+1$, and go to iteration $k$. Otherwise, set $\mathrm{UB}_{k}=\max \left\{\mathrm{UB}(H) \mid H \in Q^{k}\right\}$. Choose a rectangle $H^{k} \in Q^{k}$ such that $\mathrm{UB}\left(H^{k}\right)=\mathrm{UB}_{k}$, set $k=k+1$, and go to iteration $k$.

4.4. Convergence. In this subsection, we give the global convergence of the above algorithm. By the construction of the algorithm, when the algorithm is finite, it either finds a global optimal solution for problem $(\mathrm{P} 1(H))$ or detects that problem $(\mathrm{P} 1(H))$ is infeasible. It is also possible for the algorithm to be infinite. We will discuss this case in the following.

Denote $M^{0}=\left\{(s, t, u) \in R^{3 p} \mid 0 \leqslant s_{i} \leqslant \bar{s}_{i}, 0 \leqslant t_{i} \leqslant\right.$ $\left.\bar{t}_{i}, 0 \leqslant u_{i} \leqslant U_{i}^{0}, i \in I\right\}$. Suppose that $\widehat{M}$ denotes $M^{0}$ or a subrectangle of $M^{0}$ that is generated by the branch and bound algorithm. Then, $\widehat{M}$ may be written as

$$
\widehat{M}=\widehat{M}_{1} \times \widehat{M}_{2} \times \cdots \widehat{M}_{p}
$$

where, for any $i \in I$,

$$
\begin{aligned}
& \widehat{M}_{i} \\
& =\left\{\left(s_{i}, t_{i}, u_{i}\right) \in R^{3} \mid 0 \leqslant s_{i} \leqslant \bar{s}_{i}, 0 \leqslant t_{i} \leqslant \bar{t}_{i}, L_{i} \leqslant u_{i} \leqslant U_{i}\right\},
\end{aligned}
$$

where for each $i=1,2, \ldots, p, L_{i}, U_{i}$ are positive scalars such that $L_{i} \leqslant U_{i}$. 
If the algorithm is infinite, by the rectangular bisection, since $i \in I$ is finite, there exists an infinite sequence $\left\{M^{k}\right\}_{k=1}^{\infty}$ of rectangles in $R^{3 p}$ generated by the algorithm such that, for any $i \in I, M^{k+1} \subseteq M^{k}$ and $M^{k+1}$ is formed from $M^{k}$. By Step 1 of the rectangular bisection process, for some fixed $j_{0} \in\{i=$ $1,2, \ldots, p\}$,

$$
U_{j_{0}}^{k}-L_{j_{0}}^{k}=\max _{i \in I}\left\{U_{i}^{k}-L_{i}^{k}\right\}, \quad I=\{1,2, \ldots, p\},
$$

where $M^{k}=\left\{(s, t, u) \in R^{3 p} \mid 0 \leqslant s_{i} \leqslant \bar{s}_{i}, 0 \leqslant t_{i} \leqslant \bar{t}_{i}, L_{i}^{k} \leqslant\right.$ $\left.u_{i} \leqslant U_{i}^{k}\right\}$ for all $k$. Next, let $\left\{M^{k}\right\}_{k=1}^{\infty}$ be a sequence of rectangle of this type, and, for all $k$ and any $i \in I$, let

$$
\begin{aligned}
& M_{i}^{k} \\
& =\left\{\left(s_{i}, t_{i}, u_{i}\right) \in R^{3} \mid 0 \leqslant s_{i} \leqslant \bar{s}_{i}, 0 \leqslant t_{i} \leqslant \bar{t}_{i}, L_{i}^{k} \leqslant u_{i} \leqslant U_{i}^{k}\right\} .
\end{aligned}
$$

Lemma 9. For some subsequence $K$ of $\{1,2, \ldots\}$, the limit rectangle

$$
\begin{aligned}
M_{j_{0}}^{\infty} & =\bigcap_{k \in K} M_{j_{0}}^{k} \\
& =\left\{\left(s_{j_{0}}, t_{j_{0}}, u_{j_{0}}\right) \mid 0 \leqslant s_{j_{0}} \leqslant \bar{s}_{j_{0}}, 0 \leqslant t_{j_{0}} \leqslant \bar{t}_{j_{0}}, u_{j_{0}}=\bar{v}\right\}
\end{aligned}
$$

is rectangle in $R^{3}$ parallel to the $\left(s_{j_{0}}, t_{j_{0}}\right)$-coordinate plane.

Proof. By Lemma 5.4 in [17] and the rectangle bisection, there exists a subsequence $K$ of $\{1,2, \ldots\}$ such that

$$
\begin{gathered}
\lim _{k \in K} L_{j_{0}}^{k}=L_{j_{0}} \in R, \quad \lim _{k \in K} U_{j_{0}}^{k}=U_{j_{0}} \in R, \\
\lim _{k \in K} v_{j_{0}}^{k}=v_{j_{0}}^{*} \in\left\{L_{j_{0}}, U_{j_{0}}\right\},
\end{gathered}
$$

where $v_{j_{0}}^{k}=(1 / 2)\left(L_{j_{0}}^{k-1}+U_{j_{0}}^{k-1}\right)$. So, either $v_{j_{0}}^{*}=L_{j_{0}}$ or $v_{j_{0}}^{*}=$ $U_{j_{0}}$. Then,

$$
\begin{aligned}
M_{j_{0}}^{\infty} & =\bigcap_{k \in K} M_{j_{0}}^{k} \\
& =\left\{\left(s_{j_{0}}, t_{j_{0}}, u_{j_{0}}\right) \mid 0 \leqslant s_{j_{0}} \leqslant \bar{s}_{j_{0}}, 0 \leqslant t_{j_{0}} \leqslant \bar{t}_{j_{0}}, u_{j_{0}}=\bar{v}\right\},
\end{aligned}
$$

which is a rectangle in $R^{3}$ parallel to the $\left(t_{j_{0}}, s_{j_{0}}\right)$-coordinate plane.

Theorem 10. Suppose that the proposed algorithm is infinite, and let $\left\{M^{k}\right\}_{k=1}^{\infty}$ be a sequence of rectangles in $R^{p}$ generated by the algorithm such that, for each $k=1,2, \ldots, M^{k+1} \subset M^{k}$. Let $\psi(s, t, u)=\sum_{i=1}^{p}\left[2 u_{i} t_{i}-\left(u_{i}\right)^{2} s_{i}\right]$. Then, for some subsequence $K$ of $\{1,2, \ldots\}$,

(a) $\lim _{k \in K}\left\{U B\left(H^{k}\right)-\psi\left(s^{k}, t^{k}, u^{k}\right)\right\}=0$,

(b) any accumulation point $(\bar{t}, \bar{s}, \bar{u}, \bar{x})$ of the sequence of $\left\{\left(\bar{t}^{k}, \bar{s}^{k}, \bar{u}^{k}, \bar{x}^{k}\right)\right\}_{k=1}^{\infty}$ is a global optimal solution of problem $(P 1)$.
Proof. Consider the following.

(a) Notice that, according to (39) and (40), it follows that, for each $i \in I$,

$$
\begin{aligned}
r_{i}=h_{i}^{M_{i}}\left(u_{i}, t_{i}\right) & =\min \left\{\bar{t}_{i} u_{i}+L_{i} t_{i}-\bar{t}_{i} L_{i}, U_{i} t_{i}\right\}, \\
z_{i}=h_{i M_{i}}\left(u_{i}, s_{i}\right) & =\max \left\{L_{i} s_{i}, \bar{s}_{i} u_{i}+U_{i} s_{i}-\bar{s}_{i} U_{i}\right\},
\end{aligned}
$$

and problem $\operatorname{PR1}\left(M^{k}\right)$ may be rewritten as

$$
\begin{array}{ll}
\mathrm{UB}\left(M^{k}\right)=\max & \Psi(s, t, u) \\
\text { s.t. } \quad & t_{i}-\sqrt{f_{i}(x)} \leqslant 0, \quad-s_{i}+g_{i}(x) \leqslant 0, \\
& 0 \leqslant t_{i} \leqslant \bar{t}_{i}, \quad 0 \leqslant s_{i} \leqslant \bar{s}_{i}, \\
& L_{i} \leqslant u_{i} \leqslant U_{i}, \quad r_{i} \geqslant 0, \quad z_{i} \geqslant 0, \\
& x \in X,
\end{array}
$$

where $\Psi(s, t, u)=\sum_{i=1}^{p}\left[2 h_{i}^{M_{i}}\left(u_{i}, t_{i}\right)-K_{i} h_{i M_{i}}\left(u_{i}, s_{i}\right)-\right.$ $\left.(1 / 4) K_{i}^{2} s_{i}\right]$.

By the algorithm, since $\left\{M^{k}\right\}_{k=1}^{\infty}$ is infinite, we may choose a sequence $K$ of $\{1,2, \ldots\}$ such that, for each $k \in K$, $\mathrm{UB}\left\{M^{k}\right\} \neq-\infty$. At the same time, without loss of generality, we may assume that $\left\{M^{k}\right\}_{k \in K}$ have the properties of Lemma 9. Since for each $k \in K, \operatorname{UB}\left\{M^{k}\right\} \neq-\infty$, by the upper bounding process

$$
\Psi\left(s^{k}, t^{k}, u^{k}\right)=\operatorname{UB}\left(M^{k}\right) .
$$

By applying Lemma 9 repeatedly, we may assume that

$$
\lim _{k \in K} M^{k}=M_{1}^{\infty} \times M_{2}^{\infty} \times \cdots \times M_{p}^{\infty}=M^{\infty},
$$

where for each $i=1,2, \ldots, p, M_{i}^{\infty}$ is a rectangle in $R^{3}$ parallel to the $\left(t_{j_{0}}, s_{j_{0}}\right)$-coordinate plane. Let $Z\left(M^{k}\right)$ be the feasible domain of problem $\left(\operatorname{PR} 1\left(M^{k}\right)\right)$. For each $k \in K$,

$$
\begin{aligned}
\operatorname{UB}\left(M^{k}\right) & =\max _{(s, t, u, x) \in Z\left(M^{k}\right)} \Psi(s, t, u) \\
& \geqslant v_{M} \\
& \geqslant \psi\left(\bar{s}^{k}, \bar{t}^{k}, \bar{u}^{k}\right) \\
& \geqslant \psi\left(s^{k}, t^{k}, u^{k}\right),
\end{aligned}
$$

where the first equation follows, since $\left(s^{k}, t^{k}, u^{k}, x^{k}\right) \in$ $Z\left(M^{k}\right) \neq \emptyset$, from the definition of $\operatorname{UB}\left(M^{k}\right)$ in the upper bounding process, the first inequality follows from Step $k .2$ of the rectangle bisection algorithm and the validity of the upper bounding process, the second inequality follows because $\left(\bar{s}^{k}, \bar{t}^{k}, \bar{u}^{k}, \bar{x}^{k}\right) \in Z\left(M^{k}\right)$, and the third inequality holds by the choice of the incumbent solution in Step k.4 of algorithm. 
For each $k \in K,\left(s^{k}, t^{k}, u^{k}, x^{k}\right) \in Z\left(M^{k}\right) \subseteq Z(M)$; therefore, there is a convergence subsequence of $\left\{\left(s^{k}, t^{k}, u^{k}, x^{k}\right)\right\}_{k \in K}$, and, by (56), the limit point of this sequence lies in $M^{\infty}$. Without loss of generality, assume that

$$
\lim _{k \in K}\left(s^{k}, t^{k}, u^{k}\right)=(\bar{s}, \bar{t}, \bar{u}) \in M^{\infty} .
$$

By the continuity of $\psi(s, t, u)$ on $M$,

$$
\lim _{k \in K} \psi\left(s^{k}, t^{k}, u^{k}\right)=\psi(\bar{s}, \bar{t}, \bar{u}) .
$$

For all $k \in K,\left(s^{k}, t^{k}, u^{k}\right) \in M^{k}$, by (57),

$$
\lim _{k \in K} \Psi\left(s^{k}, t^{k}, u^{k}\right)=\psi(\bar{s}, \bar{t}, \bar{u}) .
$$

Combining (55), (57), and (58), we have

$$
\psi(\bar{s}, \bar{t}, \bar{u})=\lim _{k \in K} \mathrm{UB}\left(M^{k}\right)=\lim _{k \in K} \psi\left(s^{k}, t^{k}, u^{k}\right)=\psi(\bar{s}, \bar{t}, \bar{u}) .
$$

Since for each $k \in K, \psi\left(\bar{s}^{k}, \bar{t}^{k}, \bar{u}^{k}\right)=\sum_{i=1}^{p}\left[2 u_{i} t_{i}-\left(u_{i}\right)^{2} s_{i}\right]$, this confirms the assertion.

(b) By algorithm and (a), we obtain

$$
\lim _{k \rightarrow \infty} v^{k}=\lim _{k \rightarrow \infty}\left(\sum_{i=1}^{p}\left[2 u_{i} t_{i}-\left(u_{i}\right)^{2} s_{i}\right]\right)=v_{M} .
$$

Let $(\bar{s}, \bar{t}, \bar{u}, \bar{x})$ be an accumulation point of $\left\{\left(\bar{s}^{k}, \bar{t}^{k}, \bar{u}^{k}, \bar{x}^{k}\right)\right\}_{k=1}^{\infty}$; then for some $K \subseteq\{1,2, \ldots\}$

$$
\lim _{k \in \mathrm{K}}\left(\bar{s}^{k}, \bar{t}^{k}, \bar{u}^{k}, \bar{x}^{k}\right)=(\bar{s}, \bar{t}, \bar{u}, \bar{x}) .
$$

By (62), since $\left\{\sum_{i=1}^{p}\left[2 \bar{u}_{i}^{k} t_{i}^{k}-\left(\bar{u}_{i}^{k}\right)^{2} \bar{s}_{i}^{k}\right]\right\}_{k \in K}$ is a subsequence of $\left\{\sum_{i=1}^{p}\left[2 \bar{u}_{i}^{k} \bar{t}_{i}^{k}-\left(\bar{u}_{i}^{k}\right)^{2} \bar{s}_{i}^{k}\right]\right\}_{k=1}^{\infty}$,

$$
\lim _{k \in K}\left(\sum_{i=1}^{p}\left[2 \bar{u}_{i}^{k} \bar{t}_{i}^{k}-\left(\bar{u}_{i}^{k}\right)^{2} \bar{s}_{i}^{k}\right]\right)=v_{M} .
$$

From (63) and the continuity of the objective function of problem $(\mathrm{P}(M))$,

$$
\lim _{k \in K}\left(\sum_{i=1}^{p}\left[2 \bar{u}_{i}^{k} \bar{t}_{i}^{k}-\left(\bar{u}_{i}^{k}\right)^{2} \bar{s}_{i}^{k}\right]\right)=\sum_{i=1}^{p}\left[2 \bar{u}_{i} \bar{t}_{i}-\left(\bar{u}_{i}\right)^{2} \bar{s}_{i}\right] .
$$

From (63) and (64), we get

$$
\sum_{i=1}^{p}\left[2 \bar{u}_{i} \bar{t}_{i}-\left(\bar{u}_{i}\right)^{2} \bar{s}_{i}\right]=v_{M}
$$

Since the feasible region $Z(M)$ of problem $(\mathrm{P}(M))$ is a closed set, $(\bar{s}, \bar{t}, \bar{u}, \bar{x}) \in Z(M)$. It follows from (65) that $(\bar{s}, \bar{t}, \bar{u}, \bar{x})$ is global optimal solution for problem $(\mathrm{P}(M))$; the proof is complete.
By the algorithm, it may happen that, even after many iterations, $R_{k}$ may remain nonempty. However, by the convergence result, it follows that, for any $\epsilon>0$,

$$
\left(\mathrm{UB}_{k}-\sum_{i=1}^{p}\left[2 \bar{u}_{i}^{k-k} \bar{t}_{i}^{k}-\left(\bar{u}_{i}^{k}\right)^{2} \bar{s}_{i}^{k}\right]\right) \leqslant \epsilon
$$

will hold for $k$ sufficiently large. In practice, it is recommended that the algorithm be terminated if, for some prechosen, relatively small value of $\epsilon>0$, (66), holds. When termination occurs in this way, it is easy to show that $\bar{x}^{k}$ is a global $\epsilon$-optimal solution, and $f\left(\bar{x}^{k}\right)$ is a global $\epsilon$-optimal value for problem (LMP) in the sense that $\bar{x}^{k} \in X$ and $f\left(\bar{x}^{k}\right)+\epsilon \leqslant v$.

\section{Numerical Experiments}

To verify performance of the proposed global optimization algorithm, some test problems were implemented. The test problems are coded in $\mathrm{C}++$ and the experiments are conducted on a Pentium IV (3.06 GHZ) microcomputer.

Example 11. Consider

$$
\begin{aligned}
& v=\max \quad f(x)= \frac{-x_{1}^{2}+3 x_{1}-x_{2}^{2}+3 x_{2}+3.5}{x_{1}+1} \\
&+\frac{x_{2}}{x_{1}^{2}-2 x_{1}+x_{2}^{2}-8 x_{2}+20}, \\
& \text { s.t. } x \in X=\left\{\begin{array}{c}
2 x_{1}+x_{2} \leqslant 6, \\
3 x_{1}+x_{2} \leqslant 8, \\
x_{1}-x_{2} \leqslant 1, \\
x_{1} \geqslant 1, x_{2} \geqslant 1 .
\end{array}\right\} .
\end{aligned}
$$

Prior to initiating the algorithm, we first determine a rectangle $H^{0}=\left\{\left(u_{1}, u_{2}\right) \mid 0.9354143 \leqslant u_{1} \leqslant\right.$ $\left.1.561249,0.0769230 \leqslant u_{2} \leqslant 0.6666667\right\}$. Then, the problem (P1) is

$$
\begin{gathered}
v 1=\max \quad \varphi\left(u_{1}, u_{2}\right) \\
\text { s.t. } u \in H^{0},
\end{gathered}
$$

where

$$
\begin{array}{r}
\varphi\left(u_{1}, u_{2}\right) \\
=\max _{x \in X}\left[2 u_{1} \sqrt{-x_{1}^{2}+3 x_{1}-x_{2}^{2}+3 x_{2}+3.5}-u_{1}^{2}\left(x_{1}+1\right)\right. \\
\left.+2 u_{2} \sqrt{x_{2}}-u_{2}^{2}\left(x_{1}^{2}-2 x_{1}+x_{2}^{2}-8 x_{2}+20\right)\right] .
\end{array}
$$

Solving the linear programming $\left(\operatorname{PR} 1\left(H^{0}\right)\right)$ gets initial upper bound $\mathrm{UB}_{0}=5.793653$, and the lower bound $\mathrm{LB}_{0}=$ 3.952468 with $u_{1}=1.181421, u_{2}=0.2448056$. Set $\varepsilon=0.01$. The algorithm finds a global $\varepsilon$-optimal value 4.060819 after 23 iterations at the global $\varepsilon$-optimal solution $x^{*}=(1,1.743823)$. 
TABLE 1: Computational results of Example 12.

\begin{tabular}{lccccc}
\hline Methods & $\varepsilon$ & $\varepsilon$-optimal solution & $\varepsilon$-optimal value & Iteration number & CPU time \\
\hline$[11]$ & $1.0 E-5$ & $(1.0000,1.4142)$ & 0.4856 & 90 & $6.9329 \mathrm{~s}$ \\
Our & $1.0 E-5$ & $(1.00000,1.41422)$ & 0.48560 & 58 & $3.4545 \mathrm{~s}$ \\
\hline
\end{tabular}

TABLE 2: Computational results of Example 13.

\begin{tabular}{lccc}
\hline$n$ & $m$ & Ave. Iter & Ave. CPU (s) \\
\hline 5 & 3 & 69 & 7.8 \\
5 & 7 & 76 & 8.9 \\
5 & 10 & 84 & 9.6 \\
5 & 20 & 99 & 23.5 \\
10 & 3 & 261 & 59.6 \\
15 & 3 & 329 & 78.3 \\
\hline
\end{tabular}

Example 12 (see [11]). Consider

$$
\begin{array}{ll}
v=\max & f(x)=\frac{-2 x_{1}-x_{2}}{x_{1}+10}+\frac{-2}{x_{2}+10}, \\
\text { s.t. } \quad & -x_{1}^{2}-x_{2}^{2}+3 \leqslant 0 \\
& -x_{1}^{2}-x_{2}^{2}+8 x_{2}-14 \leqslant 0, \\
& 2 x_{1}+x_{2} \leqslant 6 \\
& 3 x_{1}+x_{2} \leqslant 8 \\
& x_{1}-x_{2} \leqslant 1, \\
& x_{1} \geqslant 1, \quad x_{2} \geqslant 1 .
\end{array}
$$

See Table 1

Example 13. In this example, we solve 6 different random instances:

$$
\begin{array}{ll}
\max & \frac{x^{T} Q_{1} x-c_{1}}{x^{T} P_{1} x}+\frac{x^{T} Q_{2} x-c_{1}}{x^{T} P_{2} x} \\
\text { s.t. } & \sum_{i=1}^{n} x_{i}=1, \\
& A x \leqslant b, \\
& 0 \leqslant x_{i} \leqslant 1, \quad i=1,2, \ldots, n,
\end{array}
$$

where $Q_{1}$ and $Q_{2}$ are negative semidefinite, while $P_{1}$ and $P_{2}$ are definite, $A$ is $m \times n$ matrix, and all elements of $Q_{1}, Q_{2}$, $P_{1}, P_{2}, A, b$ are randomly generated, whose ranges are $[0,1]$. Table 2 summarizes our computational results. In Table 2 , the following indices characterize performance in algorithm: Ave. CPU (s) is the average CPU times in seconds; Ave. Iter is average number of iterations.

\section{Conclusion}

In this paper, we present a branch and bound algorithm for solving a class of fractional programming problems $(\mathrm{P})$.
To globally solve problem (P), we first convert problem (P) into an equivalent problem (P1); then through linearization method, we obtain a convex relaxation programming problem $(\mathrm{PR} 1(H))$ of problem $(\mathrm{P} 1)$. In the algorithm, the branch and bound tree creates rectangular regions that belong to $R^{3 p}$, where $p$ is the number of ratios in the objective function of problem (P1). However, the branching process only takes place in $R^{p}$, rather than $R^{3 p}$. In addition, all subproblems that must be solved to implement the algorithm are convex programming problems, each of which is guaranteed to have an optimal solution.

\section{Conflict of Interests}

The authors declare that there is no conflict of interests regarding the publication of this paper.

\section{Acknowledgments}

Project is supported by the Ph.D. Start-Up Fund of Natural Science Foundation of Guangdong Province, China (no. S2013040012506), Project Science Foundation of Guangdong University of Finance (no. 2012RCYJ005), and the Postdoctoral Fund of Shenyang Agricultural University (no. 770212025).

\section{References}

[1] T. Ibaraki, "Parametric approaches to fractional programs," Mathematical Programming, vol. 26, no. 3, pp. 345-362, 1983.

[2] S. S. Chadha, "Fractional programming with absolute-value functions," European Journal of Operational Research, vol. 141, no. 1, pp. 233-238, 2002.

[3] T. Kuno, "A branch-and-bound algorithm for maximizing the sum of several linear ratios," Journal of Global Optimization, vol. 22, pp. 155-174, 2002.

[4] P.-P. Shen and C.-F. Wang, "Global optimization for sum of linear ratios problem with coefficients," Applied Mathematics and Computation, vol. 176, no. 1, pp. 219-229, 2006.

[5] H. P. Benson, "On the global optimization of sums of linear fractional functions over a convex set," Journal of Optimization Theory and Applications, vol. 121, no. 1, pp. 19-39, 2004.

[6] H. P. Benson, "Using concave envelopes to globally solve the nonlinear sum of ratios problem," Journal of Global Optimization, vol. 22, pp. 343-364, 2002.

[7] H. P. Benson, "Global optimization algorithm for the nonlinear sum of ratios problem," Journal of Optimization Theory and Applications, vol. 112, no. 1, pp. 1-29, 2002.

[8] C.-T. Chang, "On the posynomial fractional programming problems," European Journal of Operational Research, vol. 143, no. 1, pp. 42-52, 2002. 
[9] Y.-J. Wang and K.-C. Zhang, "Global optimization of nonlinear sum of ratios problem," Applied Mathematics and Computation, vol. 158, no. 2, pp. 319-330, 2004.

[10] R. W. Freund and F. Jarre, "Solving the sum-of-ratios problem by an interior-point method," Journal of Global Optimization, vol. 19, no. 1, pp. 83-102, 2001.

[11] P.-P. Shen, Y.-P. Duan, and Y.-G. Pei, "A simplicial branch and duality bound algorithm for the sum of convex-convex ratios problem," Journal of Computational and Applied Mathematics, vol. 223, no. 1, pp. 145-158, 2009.

[12] H. P. Benson, "Global maximization of a generalized concave multiplicative function," Journal of Optimization Theory and Applications, vol. 137, no. 1, pp. 105-120, 2008.

[13] M. Avriel, W. E. Diewart, S. Schaible, and I. Zang, Generalized Concavity, Plenum, New York, NY, USA, 1988.

[14] R. Horst and N. V. Thoai, "Decomposition approach for the global minimization of biconcave functions over polytopes," Journal of Optimization Theory and Applications, vol. 88, no. 3, pp. 561-583, 1996.

[15] R. Horst and H. Tuy, Global Optimization: Deterministic Approaches, Springer, Berlin, Germany, 1993.

[16] H. P. Benson, "On the construction of convex and concave envelope formulas for bilinear and fractional functions on quadrilaterals," Computational Optimization and Applications, vol. 27, no. 1, pp. 5-22, 2004.

[17] H. Tuy, Convex Analysis and Global Optimization, Kluwer Academic Publishers, Dordrecht, The Netherlands, 1998. 


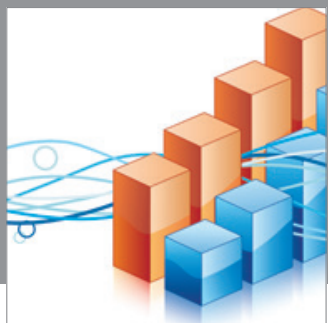

Advances in

Operations Research

mansans

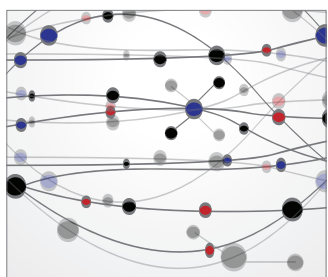

The Scientific World Journal
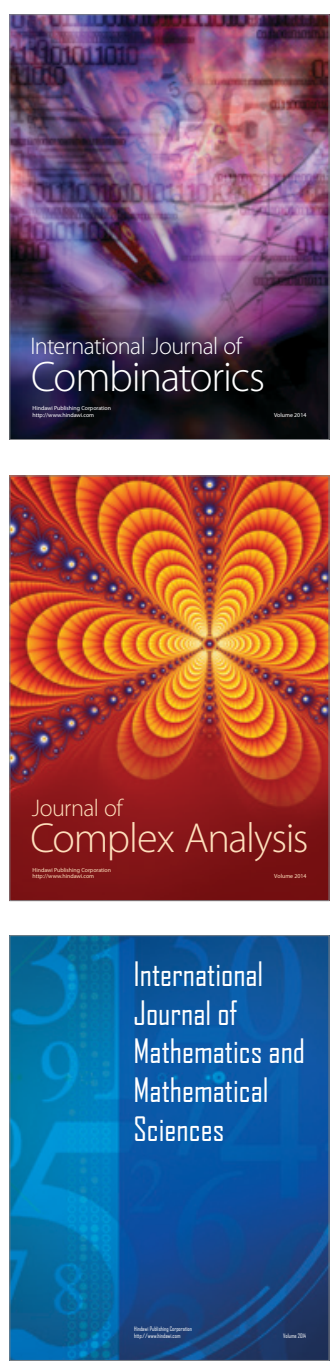
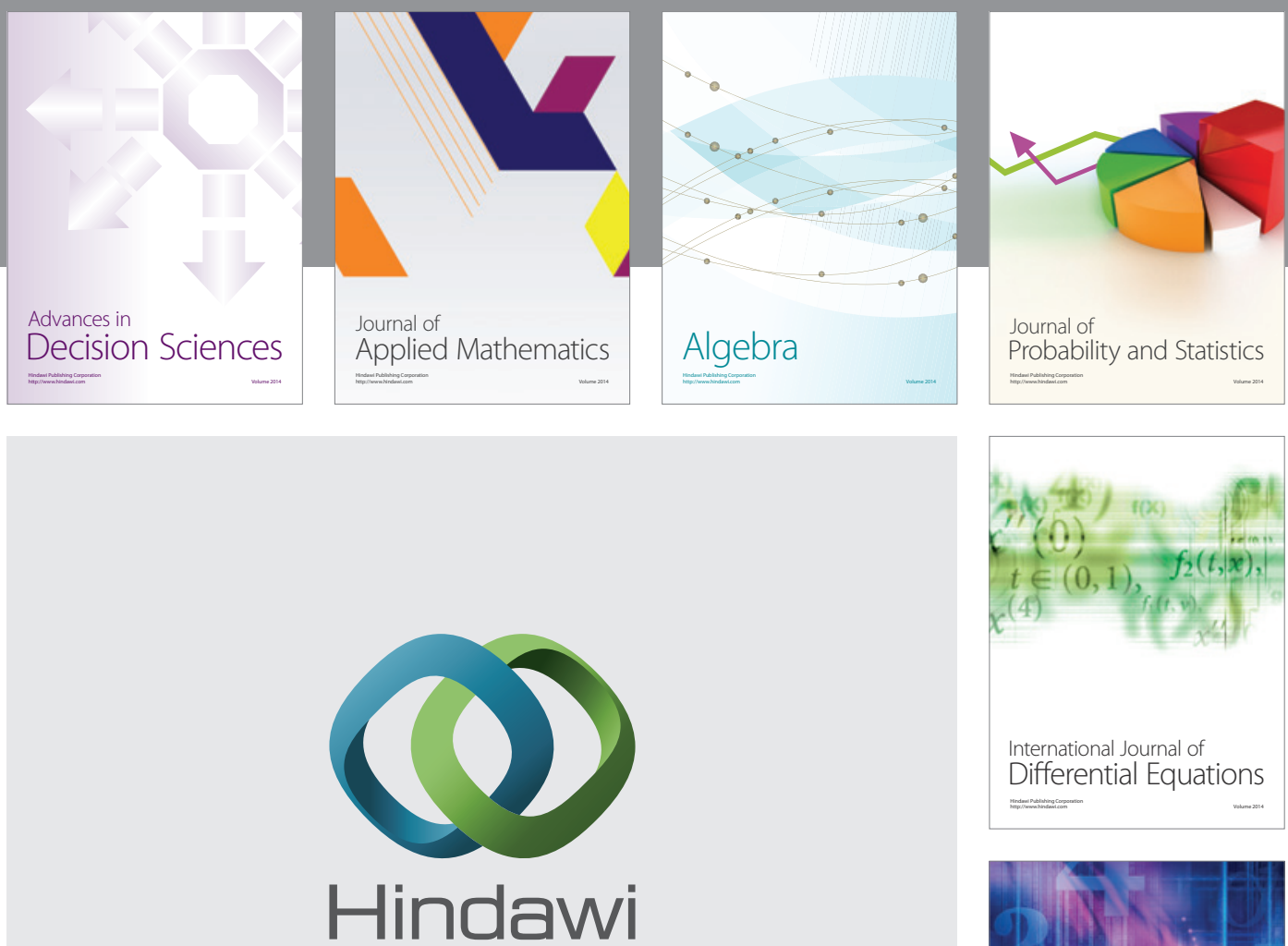

Submit your manuscripts at http://www.hindawi.com
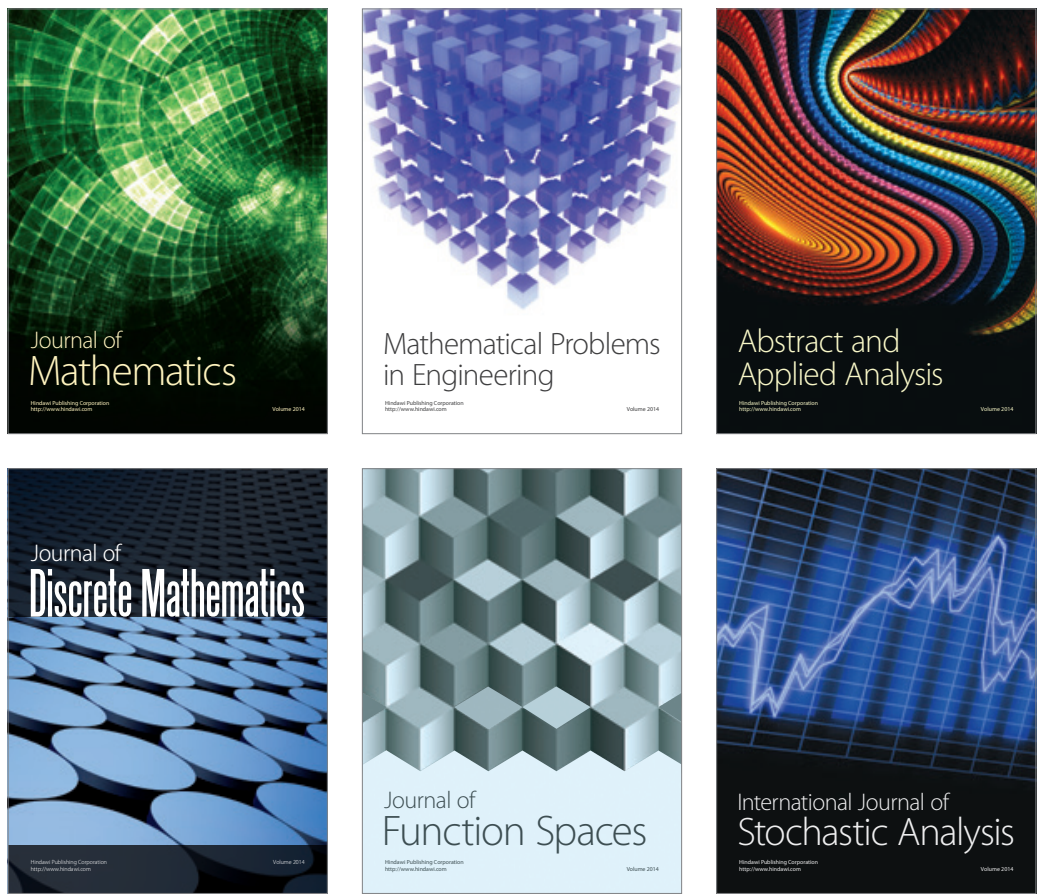

Journal of

Function Spaces

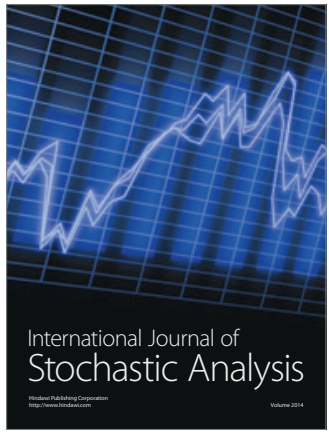

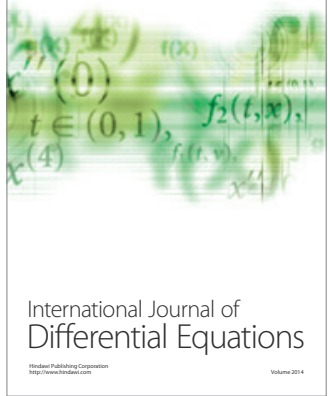
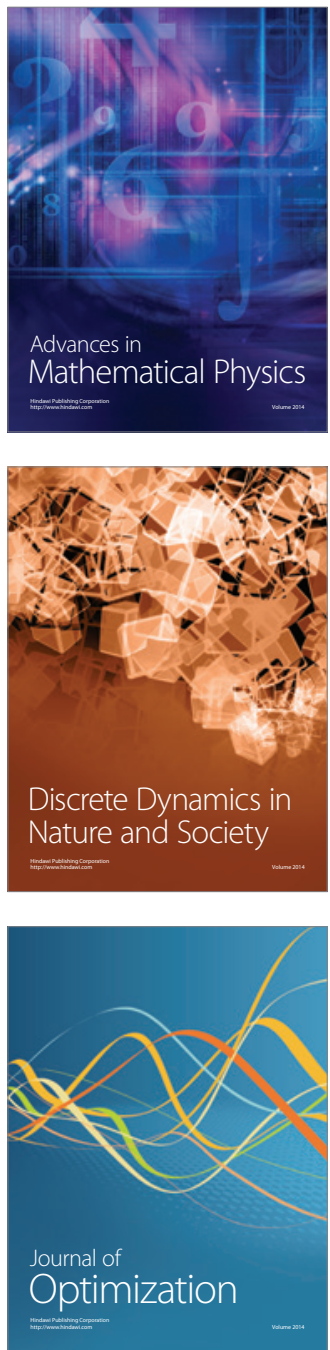